\title{
The Effect of Theracurmin on Cognitive Function in an Older Patient with Chemobrain
}

This article was published in the following Dove Press journal:

Clinical Interventions in Aging

\author{
Neziha Erken (ID) \\ Fatma Sena Dost Gunay (D) \\ Pinar Soysal (iD ${ }^{2}$ \\ Ahmet Turan Isik (D) \\ 'Department of Geriatric Medicine, \\ Faculty of Medicine, Dokuz Eylul \\ University, Izmir, Turkey; ${ }^{2}$ Department of \\ Geriatric Medicine, Bezmialem Vakif \\ University, Faculty of Medicine, Istanbul, \\ Turkey
}

\begin{abstract}
Chemobrain is one of the problems that may arise during or after treatment and there is currently no specific treatment for this condition. Our case was a 76-year-old female patient who presented to our clinic with complaints of forgetfulness that did not affect daily living activities for the last year. Breast cancer was diagnosed in 2013 and she has been receiving anastrozole treatment for 6 years after local mass excision surgery and radiotherapy. After a comprehensive geriatric evaluation, cognitive impairment due to systemic cancer therapy was detected and treatment was started with Theracurmin $90 \mathrm{mg}$ twice a day therapy. After 3-months of Theracurmin therapy, she had no cognitive improvement during the follow-up. This case report demonstrated that Theracurmin treatment may be a new option for chemobrain.
\end{abstract}

Keywords: chemobrain, Theracurmin, elderly, cognitive impairment, cancer

\section{Background}

Both cancer and cognitive impairment are common in older people. Anti-cancer treatments may improve the likelihood of long-term survival after cancer diagnosis, while adversely affecting the cognitive function of patients, which is called chemotherapy-related cognitive impairment or chemobrain. ${ }^{1,2}$ Chemobrain can occur during or after chemotherapy and represents a concern for many patients with cancer, which can affect cognitive abilities including attention, memory, executive functions, language, visuospatial skills, and processing speed. ${ }^{3}$ Furthermore, cancer, itself, can lead to cognitive impairment as well as anti-cancer therapies. Among these therapies, aromatase inhibitors, agents for early-stage hormone receptorpositive breast cancer, may also be the cause of chemobrain by acting on estrogen receptors that modulate the expression of Apolipoprotein $\mathrm{E}$ and the estrogen gene in important brain regions, such as the hippocampus, prefrontal cortex, and amygdala, for cognitive functions. ${ }^{1,4}$ The prevalence was estimated to be between $12 \%$ and $78 \%$ of breast cancer patients after initiation of chemotherapy, and cognitive impairment includes various areas such as executive function, memory, psychomotor speed, and attention. ${ }^{2,3}$ Additionally, the severity of cognitive impairment is characteristically mild to moderate in nature, but even mild impairment can severely affect the quality of life, especially in older people. Chemobrain cannot only adversely affect daily living activities, access to medical care facilities, and quality of care, but together with the cancer-induced catabolic process also increases mortality. ${ }^{1-3}$ In addition, although many pharmacological agents such as psychostimulants, acetylcholinesterase inhibitors, N-methyl-d- aspartate receptor
Correspondence: Ahmet Turan Isik Unit for Brain Aging and Dementia, Department of Geriatric Medicine, School of Medicine, Dokuz Eylul University, Izmir, Turkey

Tel +902324124341

Fax +902324124349

Email atisik@yahoo.com 
antagonists and ginkgo Biloba have been tried for chemobrain treatment with promising results, there is no cure for chemobrain yet. $^{5}$

Curcumin, commonly used as a spice, food additive or dietary pigment in Asia and the Middle East, has several beneficial effects, such as nephroprotective, anti-cancer, antithrombotic, anti-ischemic, anti-hyperglycemic, and antirheumatic effects as well as cognitive enhancer effects. ${ }^{6,7}$ Moreover, it has been reported that it may improve memory and attention in non-demented elderly patients. ${ }^{8}$

In this report, we present an older woman with chemobrain treated with Theracurmin in our geriatric outpatient clinic.

Additionally, the written informed consent for publication of her clinical details was provided.

\section{Case Presentation}

A 76-year-old woman was referred to our geriatric outpatient clinic due to memory loss. The onset of symptoms had begun about a year earlier, and forgetfulness progressed slowly, and within a year she began to forget short-term events, the names of her friends, and, rarely, things on the stove.

The medical history of the patient revealed that she had estrogen receptor positive breast cancer in 2013 and therefore underwent a local mass excision, followed by 32 cycles of local radiotherapy. She has never received intravenous or oral chemotherapy, but only used hormonal therapy, which was $1 \mathrm{mg}$ anastrozole per day for 5 years. She had received metformin $1000 \mathrm{mg} /$ bid due to Type 2 Diabetes Mellitus for 10 years. Comprehensive geriatric evaluation was performed for the patient. ${ }^{9}$ Physical examination revealed normal mood, recall, deterioration in language and abstracting, and her daily living activities were preserved. During the family interview; her husband said that she was able to perform daily living activities but suffered from forgetfulness, and difficulty in concentrating and multitasking. Accordingly, her MOCA (MoCA version 7.1) score was 22. The clinical and biochemical findings are summarized in Table 1. The neuroimaging study showed mild chronic ischemic changes and age-compatible atrophy.

In the light of clinical, laboratory and brain imaging assessment and family interview situation, she met mild cognitive impairment (MCI) diagnostic criteria. ${ }^{10}$ Additionally, given the fact that she has been using anastrozole for 5 years due to breast cancer and other possible causes of her symptoms have been excluded, a chemobrain diagnosis was established, and Theracurmin was initiated
Table I Laboratory and Neurocognitive Tests Score Follow-Up

\begin{tabular}{|l|l|l|}
\hline Parameters & Baseline & Follow-Up \\
\hline Biochemical Evaluation & & \\
Fasting glucose (mg/dL) & 88 & $7 I$ \\
Hemoglobin (mg/dL) & 11.8 & 12 \\
Glomerular filtration rate (CKD-EPI) & 56 & 54 \\
Sodium (mmol/L) & 136 & 140 \\
Potassium (mmol/L) & 4.7 & 4.7 \\
Vitamin BI2 (pg/mL) & 507 & 687 \\
Vitamin D (ng/mL) & 47.1 & 22.5 \\
Folic acid (ng/mL) & 23 & 17 \\
Thyroid stimulating hormone (m[IU]/L) & 1.7 & 2.3 \\
Free T4 (ng/dL) & 0.95 & 0.93 \\
HbAlc (\%) & 6.7 & 6.8 \\
\hline Comprehensive Geriatric Assessment & & \\
Barthel ADL & 100 & 100 \\
Instrumental ADL & 20 & 22 \\
GDS & 1 & 1 \\
CDR & 0.5 & 0 \\
MoCA score & 22 & 26 \\
Visuospatial/executive & 4 & 5 \\
Naming & 3 & 3 \\
Attention & 6 & 6 \\
Language & 1 & 2 \\
Abstraction & 1 & 1 \\
Delayed recall & 1 & 3 \\
Orientation & 6 & 6 \\
\hline
\end{tabular}

Abbreviations: GDS, Geriatric Depression Scale (0-15); BADL, Basic Activities of Daily Living (0-100); CDR, Clinical Dementia Rating Scale (0-3); IADL, Instrumental Activities of Daily Living (0-23); MoCA, Montreal Cognitive Assessment Scale (0-30).

at a dosage of $90 \mathrm{mg}$ twice a day, as a complementary treatment due to many aforementioned advantages.

\section{Outcome and Follow-Up}

During the follow-up visit, her MOCA score was 26 (especially improvement in delayed recall) after 3 months of Theracurmin, with improved symptoms (Table 1).

\section{Discussion}

Theracurmin treatment was reported to improve cognitive impairment in an older patient with chemobrain.

Today, with advances in cancer treatments, survival is increased, making the treatment side effects more important, especially in older people. ${ }^{11}$ Chemobrain is one of the problems that may arise during or after treatment, 5,12 which is important for both the patient and the caregivers to affect the quality of life and the increase in health expenditures. ${ }^{1,12}$ However, the underlying mechanisms of chemobrain are not completely understood yet, it is 
considered that many factors are responsible and anticancer drugs related damage on CNS progenitor cells, neurogenesis, and oligodendrocytes may play an important role in the pathogenesis. ${ }^{1-3,12}$ In addition, to the best of our knowledge, no pharmacological treatment for the disease has so far been developed.

Curcumin has been used since 1815 as a food, coloring agent, and traditional medicine and lastly as a promising anticancer drug with a natural polyphenol derived from turmeric and clinical trials are continuing or completed for different cancer types, including breast, pancreatic and colorectal cancers, and multiple myeloma. ${ }^{6,7}$ A recent study has shown that Theracurmin, a safe and active kind of curcumin, may improve memory and attention in non-demented older people. $^{8}$ This improvement may be associated with the cognitive enhancement, anti-inflammatory, antioxidant, antiamyloid and possible anti-tau properties of Theracurmin. ${ }^{6-8}$

In our case, 3-month Theracurmin therapy improved the cognitive symptoms related to chemobrain in an older adult. Under Theracurmin therapy, she showed improvements in executive and language cognitive domains which are the two main affected cognitive domains in chemobrain. These results are important as Theracurmin might be a treatment option for chemobrain in the future. In addition, other positive effects of Theracurmin such as antidiabetic and cardio protection may have contributed to this improvement. ${ }^{5,6}$

The potential negative outcomes of chemobrain have adverse effects on the management of older cancer patients; therefore, cognitive screening tests should be part of routine assessment for these patients, and if necessary, they should be referred to a geriatrician for further evaluation. Furthermore, due to its potential positive effects on cognitive function as well as anti-cancer effects, Theracurmin might be a new treatment option for chemobrain, but further studies are needed.

\section{Disclosure}

The authors report no conflicts of interest in this work.

\section{References}

1. Moore HC. An overview of chemotherapy-related cognitive dysfunction, or 'chemobrain'. Oncology (Williston Park). 2014;28(9):797-804.

2. Loh KP, Janelsins MC, Mohile SG, et al. Chemotherapy-related cognitive impairment in older patients with cancer. $J$ Geriatr Oncol. 2016;7(4):270-280. doi:10.1016/j.jgo.2016.04.008

3. Wang XM, Walitt B, Saligan L, Tiwari AF, Cheung CW, Zhang ZJ. Chemobrain: a critical review and causal hypothesis of link between cytokines and epigenetic reprogramming associated with chemotherapy. Cytokine. 2015;72(1):86-96. doi:10.1016/j.cyto.2014.12.006

4. Srivastava RA, Bhasin N, Srivastava N. Apolipoprotein E gene expression in various tissues of mouse and regulation by estrogen. Biochem Mol Biol Int. 1996;38(1):91-101.

5. PJC VL, Klaver KM, Wefel JS, Sitskoorn MM, Schagen SB, Gehring K. Interventions for cognitive problems in adults with brain cancer: a narrative review. Eur $J$ Cancer Care (Engl). 2019;28(3):e13088. doi:10.1111/ecc.13088

6. D'Cunha NM, Seddon N, Mellor DD, et al. Curcumin for cognition: is it just hype, based on current data? Adv Nutr. 2019;10(1):179-181. doi:10.1093/advances/nmy066

7. Deguchi A. Curcumin targets in inflammation and cancer. Endocr Metab Immune Disord Drug Targets. 2015;15(2):88-96. doi:10.2174/ 1871530315666150316120458

8. Small GW, Siddarth P, Li Z, et al. Memory and brain amyloid and tau effects of a bioavailable form of curcumin in non-demented adults: a double-blind, placebo-controlled 18-month trial. Am J Geriatr Psychiatry. 2018;26(3):266-277. doi:10.1016/j.jagp.2017.10.010

9. Unutmaz GD, Soysal P, Tuven B, Isik AT. Costs of medication in older patients: before and after comprehensive geriatric assessment. Clin Interv Aging. 2018;13:607-613. doi:10.2147/CIA.S159966

10. Petersen RC, Lopez O, Armstrong MJ, et al. Practice guideline update summary: mild cognitive impairment: report of the guideline development, dissemination, and implementation subcommittee of the American Academy of Neurology. Neurology. 2018;90(3):126-135. doi:10.1212/WNL.0000000000004826

11. Lange M, Rigal O, Clarisse B, et al. Cognitive dysfunctions in elderly cancer patients: a new challenge for oncologists. Cancer Treat Rev. 2014;40(6):810-817. doi:10.1016/j.ctrv.2014.03.003

12. Mandelblatt JS, Stern RA, Luta G, et al. Cognitive impairment in older patients with breast cancer before systemic therapy: is there an interaction between cancer and comorbidity? J Clin Oncol. 2014;32 (18):1909-1918. doi:10.1200/JCO.2013.54.2050
Clinical Interventions in Aging

\section{Publish your work in this journal}

Clinical Interventions in Aging is an international, peer-reviewed journal focusing on evidence-based reports on the value or lack thereof of treatments intended to prevent or delay the onset of maladaptive correlates of aging in human beings. This journal is indexed on PubMed Central, MedLine, CAS, Scopus and the Elsevie
Bibliographic databases. The manuscript management system is completely online and includes a very quick and fair peer-review system, which is all easy to use. Visit http://www.dovepress.com/ testimonials.php to read real quotes from published authors. 